Tropospheric temperature series from satellites

Arising from: Q. Fu et al. Nature 429, 55-58 (2004)

T here has been considerable debate about changes in the temperature of the troposphere $^{1}$ measured using the Microwave Sounding Unit (MSU) instrument $^{2,3}$ or radiosondes ${ }^{4,5}$. Fu et al. ${ }^{6}$ linearly combine time series from two MSU channels to estimate vertically integrated $850-300-\mathrm{hPa}$ temperatures and claim consistency between surface and free-troposphere warming for one MSU record. We believe that their approach overfits the data, produces trends that overestimate warming and gives overly optimistic uncertainty estimates. There still remain large differences between observed tropospheric temperature trends and those simulated by a climate model.

Fu et al. ${ }^{6}$ linearly combine MSU channels 2 and 4 using coefficients estimated from linear regression on a single, monthly mean radiosonde data set to create an effective weighting function that minimizes the effect of the stratosphere. For this approach to be valid on all space and time scales, the structure of stratospheric temperature variability must be stationary; this is not the case in reality. For example, the quasi-biennial oscillation ${ }^{7}$ has a temperature response of more than $1 \mathrm{~K}$ above pressures of $100 \mathrm{hPa}$, where the weightings of Fu et al. ${ }^{6}$ are negative, but little signal below $100 \mathrm{hPa}$, where the weightings are positive. Fu et al. ${ }^{6}$ will therefore alias an inverse quasibiennial oscillation signal into the tropical tropospheric record - something not apparent in radiosonde observations.

$\mathrm{Fu}$ et al. trained and tested both their channel- 2 and -4 coefficients on the same radiosonde $\mathrm{data}^{5}$, which can give false agreement and overfitting ${ }^{8}$. They found a global-average trend difference between their estimated value and actual 850-300hPa temperatures $\left(T_{850-300}\right)$ of $0.001 \mathrm{~K}$ per decade. We believe that this result is misleading: their statistical model could have been independently confirmed by at least one other vertically resolved radiosonde data set $^{4}$, a reanalysis ${ }^{9}$, a climate model forced with observed sea surface temperatures and anthropogenic and natural forcings ${ }^{10}$ or a coupled climate model forced with anthropogenic and natural forcings ${ }^{11}$. We did this for tropical trends (Table 1). Discrepancies between tropical trends computed using the method of $\mathrm{Fu}$ et al. ${ }^{6}$ $\left(T_{\text {fjws }}\right)$ and tropical $T_{850-300}$ trends range from -0.02 to $0.06 \mathrm{~K}$ per decade, with rootmean-square values ranging from 0.03 to $0.09 \mathrm{~K}$. Except for HadRT2.1s, the $T_{2 \mathrm{LT}}$ trend (where $T_{2 \mathrm{LT}}$ is a synthetic channel for lowermiddle troposphere) is a better estimate of the $T_{850-300}$ trends than $T_{\text {fjws }}$ trends. These $T_{\text {fjws }}$ trends are generally larger than the $T_{850-300}$ trends, suggesting that the approach of Fu et al. has a warm bias.

Trend discrepancies, and root-meansquare values, are smaller when $T_{\text {fjws }}$ is compared with 1,000-100-hPa temperatures $\left(T_{1,000-100}\right)$, with less evidence of systematic bias (Table 1). This is probably because of the form of the effective weighting function. However, there still exist differences of -0.01 to $0.02 \mathrm{~K}$ per decade between $T_{1,000-100}$ and $T_{\text {fjws }}$ trends - about $10 \%$ of the observed surface tropical warming.

Average tropospheric temperature trends derived from an ensemble of coupled atmosphere-ocean model simulations are similar to those in the atmosphere-only case (Table 1). However, tropospheric trend

ranges in the coupled simulations are larger than the atmosphere-only case and so are consistent with that estimated from one processing of the MSU record ${ }^{3}$. This demonstrates that ignoring observed changes in sea surface temperature leads to a weaker test of model-data consistency.

We re-estimated the channel-2 and -4 coefficients of Fu et al. ${ }^{6}$ using HadRT2.1s (ref. 4), rather than the radiosonde data set of ref. 5. Our coefficients differ from those of $\mathrm{Fu}$ et al. and are sensitive to the choice of training period, with a total uncertainty of the order of $10 \%$ for global and tropical coefficients, corresponding to a trend uncertainty of 0.01 to $0.02 \mathrm{~K}$ per decade.

Although the approach of Fu et al. is novel, independent data indicate that it contains significant uncertainty. HadAM3 and the GISS model ${ }^{12}$ forced with observed sea surface temperatures and forcing reconstructions show significantly greater warming than all 'observed' data sets. To resolve differences between models and observations requires good experimental design and process-based studies using physical understanding.

Simon Tett ${ }^{*}$, Peter Thorne $\dagger$

${ }^{*}$ Met Office, Hadley Centre (Reading Unit),

Meteorology Building, University of Reading,

Reading RG6 6BB, UK

e-mail: simon.tett@metoffice.gov.uk

$\dagger$ Met Office, Hadley Centre, Fitzroy Road,

Exeter EX1 3PB, UK

doi:10.1038/nature03208

1. Seidel, D. J. et al. J. Clim. 17, 2225-2240 (2004).

2. Christy, J. R., Spencer, R. W., Norris, W. B., Braswell, W. D. \& Parker, D. E. J. Atmos. Oceanic Tech. 20, 613-629 (2003).

3. Mears, C. A., Schabel, M. C. \& Wentz, F. J. J. Clim. 16, 3650-3664 (2003).

4. Parker, D. E. et al. Geophys. Res. Lett. 24, 1499-1502 (1997).

5. Lanzante, J. R., Klein, S. A. \& Seidel, D. J. J. Clim. 16, 224-240 (2003).

6. Fu, Q., Johanson, C. M., Warren, S. G. \& Seidel, D. J. Nature 429, 55-58 (2004).

7. Collimore, C. C., Martin, D. W., Hitchman, M. H., Huesmann, A. \& Waliser, D. E. J. Clim. 16, 2552-2568 (2003).

8. Von Storch, H. \& Zweirs, F. W. in Statistical Analysis in Climate Research 404-405 (Cambridge Univ. Press, Cambridge, 1999).

\begin{tabular}{|c|c|c|c|c|c|c|c|}
\hline Data source & $\mathrm{T}_{4}$ & $\mathrm{~T}_{2}$ & $\mathrm{~T}_{\text {fiws }}$ & $\mathrm{T}_{2 L T}$ & $T_{850-300}$ & $\mathrm{~T}_{1,000-100}$ & Surface \\
\hline $\begin{array}{l}\text { MSU (Remote } \\
\text { Sensing Systems }{ }^{3} \text { ) }\end{array}$ & -0.35 & 0.12 & 0.18 & & & & 0.13 \\
\hline $\begin{array}{l}\text { MSU (University of } \\
\text { Alabama, Huntsville }{ }^{2} \text { ) }\end{array}$ & -0.39 & 0.05 & 0.10 & 0.00 & & & 0.13 \\
\hline Radiosonde (HadRT2.1 $\mathrm{s}^{4}$ ) & -0.60 & -0.08 & -0.02 & 0.03 & $0.00(0.09)$ & $-0.03(0.07)$ & 0.13 \\
\hline $\begin{array}{l}\text { ERA40 reanalysis } \\
(1 \text { Dec } 1978-1 \text { Dec } 2001)^{9}\end{array}$ & -0.20 & 0.06 & 0.09 & 0.02 & $0.03(0.07)$ & $0.10(0.02)$ & 0.10 \\
\hline HadAM3 ${ }^{10}$ Model average & -0.29 & 0.19 & 0.25 & 0.20 & $0.22(0.03)$ & $0.23(0.02)$ & 0.14 \\
\hline Smallest trend & -0.31 & 0.17 & 0.22 & 0.18 & 0.21 & 0.21 & 0.12 \\
\hline Largest trend & -0.26 & 0.22 & 0.29 & 0.23 & 0.25 & 0.27 & 0.15 \\
\hline HadCM3 ${ }^{11}$ Model average & -0.45 & 0.16 & 0.23 & 0.21 & $0.21(0.03)$ & $0.22(0.01)$ & 0.16 \\
\hline Smallest trend & -0.42 & 0.07 & 0.13 & 0.12 & 0.12 & 0.13 & 0.10 \\
\hline Largest trend & -0.51 & 0.24 & 0.32 & 0.28 & 0.30 & 0.30 & 0.21 \\
\hline
\end{tabular}

Tropical $\left(30^{\circ} \mathrm{S}-30^{\circ} \mathrm{N}\right.$ ) trends (K per decade) are shown for deep-layer temperatures for 1 December 1978 to 1 December 2002. For the non-satellite data sets, static weighting functions were used to estimate synthetic Microwave Sounding Unit (MSU) equivalents. $T_{\text {fwi }}$ is derived for each data set by applying the Fu et al. published coefficients to the $T_{2}$ and $T_{4}$ data. All data were zonally averaged, then cosine-weighted and least-square estimates of the linear trends computed from annual-mean data. For HadRT2.1s, Indian data were removed from the analysis. Also shown are the logarithms of the pressure-weighted 850-300-hPa temperatures ${ }^{1}\left(T_{850-300}\right)$ and of the pressure-weighted 1,000-100-hPa temperatures $\left(T_{1,000-100}\right)$; the root-mean-square of the annual-mean differences between those and $T_{\text {fiws }}$ is shown in brackets. Surface trends are from data averaged over land and ocean. For ERA40, we used two-metre temperatures over land and sea surface temperatures over the oceans. Surface temperatures from HadCRUT2v are used for RSS, UAH and HadRT2.1s. For the two model ensembles, the average, largest and smallest trends are shown. The difference between largest and smallest gives an indication of uncertainty in the ensemble average. The coupled (HadCM3) and atmosphere-only (HadAM3) simulations differ in their forcings, with the main differences being a correction of an error in ozone loss and changes to the sulphur cycle in the HadAM3 simulations. The HadAM3 (HadCM3) ensemble consists of six (four) simulations.

9. Uppala, S. in Proc. Workshop Reanalysis 5-9 November 2001 1-10 (European Centre for Medium-range Weather Forecasting, Reading, 2003).

10. Pope, V. D., Gallani, M. L., Rowntree, P. R. \& Stratton, R. A. Clim. Dyn. 16, 123-146 (2000).

11. Stott, P. et al. Science 290, 2133-2137 (2000).

12. Hansen, J. et al. J. Geophys. Res. 107, doi:10.1029/2001JD001143 (2002).

Reply: Fu et al. reply to this communication

(doi:10.1038/nature03210).

\section{Atmospheric science}

\section{Stratospheric cooling} and the troposphere

S atellite observations of tropospheric temperatures seem to show less warming than surface temperatures, contrary to physical predictions ${ }^{1}$. Fu et al. ${ }^{2}$ show that statistical correction for the effect of stratospheric cooling brings the satellite-based 